\title{
Laparoscopic Colectomy for Caecal Cancer on the 8th Day After Acute Myocardial Infarction: A Case Report
}

Yuki Tateno ( $\nabla$ ytateno13044@gmail.com )

Tama Southern Region Hospital: Tama Nanbu Chiiki Byoin https://orcid.org/0000-0001-7825-8392

Yasuaki Kanada

Tama Nanbu Chiiki Byoin

Takahiro Hayashi

Tama Nanbu Chiiki Byoin

Ken-ichiro Hataji

Tama Nanbu Chiiki Byoin

\section{Case report}

Keywords: Anterior wall myocardial infarction, Emergency treatment, Laparoscopic surgery

Posted Date: April 21st, 2021

DOI: https://doi.org/10.21203/rs.3.rs-428669/v1

License: (c) (i) This work is licensed under a Creative Commons Attribution 4.0 International License.

Read Full License 


\section{Abstract}

\section{Background}

Abdominal surgery immediately after acute myocardial infarction is associated with a high risk of intraoperative complications and postoperative cardiac complications. It is recommended that elective surgery be postponed for 6 months after acute myocardial infarction. However, for oncological emergencies due to tumour bleeding, postponing surgery could cause tumour growth or metastasis, making early surgery preferable. There is no established evidence on how surgery can be performed immediately after acute myocardial infarction, while minimising the risk.

\section{Case presentation}

Our case involved an 85-year-old Asian man who underwent laparoscopic ileocaecal resection for haemorrhagic caecal cancer on the 8th day after acute myocardial infarction, with good outcomes.

\section{Conclusion}

Laparoscopic surgery can be an effective method compared to open surgery immediately after acute myocardial infarction with regard to preventing intraoperative complications and postoperative cardiac complications.

\section{Background}

Abdominal surgery immediately after the onset of acute myocardial infarction is a high-risk procedure, and according to the ASA guidelines, elective surgery should be postponed for 6 months [1]. The high risk is due to stress from general anaesthesia and surgical invasion which could increase cardiac complications such as recurrent myocardial ischaemia, cardiac rupture, fatal arrhythmia and acute heart failure [1-3]. In addition, anticoagulant therapy is usually administered immediately after PCl, which may increase the risk of bleeding during surgery [1].

However, there may be abdominal emergencies in which abdominal surgery immediately after $\mathrm{PCl}$ is unavoidable. Diseases requiring emergency surgery such as colonic perforation with severe peritonitis are common. In addition, oncological emergencies such as tumour bleeding are emergency situations, and if surgery is postponed, the tumour may grow or metastasise; thus, early surgery is desired.

However, there is no established evidence on how to perform abdominal surgery immediately after acute myocardial infarction while minimising the risk.

We performed laparoscopic ileocaecal resection for bleeding caecal cancer 8 days after acute anterior wall myocardial infarction, with good outcomes. Laparoscopic surgery is an effective method for reducing the risk of perioperative cardiac complications and surgical risk. 


\section{Case Presentation}

An 85-year-old Asian man visited our hospital on emergency with a complaint of chest pain. The patient was conscious, and the vital signs on admission were as follows: body temperature $35.8^{\circ} \mathrm{C}$, blood pressure $157 / 87 \mathrm{mmHg}$, pulse $79 / \mathrm{min}$ and SpO2 $100 \%$ on room air. ST elevation was observed on V1-V5 on the electrocardiogram, and blood tests yielded normal values except for troponin I which was elevated to $33.0 \mathrm{ng} / \mathrm{L}$; thus, acute myocardial infarction was suspected. The patient underwent emergency coronary angiography, which showed \# $6100 \%$ stenosis, and PCl was performed on the same site. A drug-eluting stent (Xience Skypoint) was placed, and there was improvement from TIMI 0 to 3 (Fig.1).

After the PCl, the patient was admitted to the intensive care unit. A blood sampling test about 6 hours after the admission showed an increase in CK7726 (CK-MB511). Echocardiography showed severe hypokinesis of the anterior wall and a decrease in ejection fraction, which is an indicator of cardiac function, to $35 \%$.

Oral antiplatelet drugs such as bufferin and clopidogrel were started, and 10,000 units of heparin per day was also administered. Heparin was discontinued on the 2 nd day after the intensive care unit admission because of melaena, which still persisted on the 3rd day. To identify the cause of the melaena, emergency lower endoscopy and simple abdominal CT examination were performed. Enhanced CT could not be performed because of renal dysfunction. The lower endoscopy revealed a type 2 tumour of about $40 \mathrm{~mm}$ in the caecum (Fig.2), and biopsy revealed moderately differentiated adenocarcinoma, leading to a diagnosis of caecal cancer. Although the bleeding had stopped at the time of observation, the tumour was fragile and we considered that bleeding could easily recur. CT showed swelling in the regional lymph node (Fig.3), which was suspected to be metastasis, but no distant metastasis was found.

The patient was at risk of melaena if he started eating; however, the antiplatelet drug could not be stopped because of the recent myocardial infarction. Therefore, we considered it was risky for the patient to start eating while taking antiplatelet drugs.

It was decided that the caecal cancer would be surgically resected. From the 6th day after admission, clopidogrel was discontinued and 10,000 units per day of heparin was started on the same day. Heparin was terminated at midnight on the day of surgery, but bufferin was continued. On the 8th day after admission, laparoscopic ileocaecal resection with D3 lymph node dissection, and functional end-to-end anastomosis of the ileum and ascending colon were performed. A 12-mm port was inserted into the navel for pneumoperitoneum, a 12-mm port was inserted above the pubis and 5-mm ports were inserted into the lower right abdomen, upper left abdomen and lower left abdomen. Pneumoperitoneum was achieved with carbon dioxide. A small laparotomy of $5 \mathrm{~cm}$ was placed on the umbilical region to remove the sample and perform anastomosis. The operation time was 2 hours and 13 minutes, and the amount of bleeding was $35 \mathrm{ml}$. Type 2 lesions were found in the resected caecum as preoperatively diagnosed (Fig.4). The final pathological examination revealed stage II cancer. Immediately after the procedure, the patient was managed in the intensive care unit, and the pain was treated with opioids. The patient was moved to the general ward on POD1. The postoperative course was uneventful, and he started eating 
from POD1 and resumed all drugs as in the preoperative period, including clopidogrel. He was discharged without complications on POD7. Two months after the operation, there was no recurrence of the tumour and no cardiac complications were observed.

\section{Discussion And Conclusions}

We performed laparoscopic ileocaecal resection for haemorrhagic caecal cancer, which was identified because of melaena, on the 8th day after acute myocardial infarction, and we obtained good outcomes. To our knowledge, no previous article has reported that laparoscopic surgery was performed at such an early period after acute myocardial infarction.

Surgery immediately after an acute myocardial infarction should be avoided [1]. However, in this case, the patient could not start eating because of melaena, and there was no prospect of discharge from the hospital. Since the cause of the bleeding was a malignant tumour and there was concern that the tumour would progress if surgery was postponed, surgery was unavoidably performed immediately after myocardial infarction.

There are cases in which abdominal surgery must be performed immediately after myocardial infarction. Regarding surgery for oncological emergencies caused by bleeding from colorectal cancer, like in the current case, we consider that laparoscopic surgery has many advantages over open surgery for four reasons from the viewpoint of preventing intraoperative complications and postoperative cardiac complications.

First, laparoscopic surgery has a lower risk of bleeding than open surgery. Surgery immediately after acute myocardial infarction often requires the continuation of antiplatelet drugs [1], with the attendant risk of increased bleeding. In the current case as well, clopidogrel was replaced with heparin which was discontinued on the day of surgery, but bufferin was continued and surgery was performed. Laparoscopic surgery causes less abdominal wall destruction and generally tends to cause less bleeding than open surgery [4]. It has been reported that compared to open surgery, laparoscopic gastrointestinal surgery for patients receiving antiplatelet or anticoagulant drugs can be safely performed without increasing the risk of bleeding [5]. In addition, a small amount of bleeding precludes the use of a large amount of fluid replacement or blood transfusion which can lead to congestive heart failure. In this case, the intraoperative bleeding was as small as $35 \mathrm{ml}$, and blood transfusion was not required.

The second reason is that there is less pain with laparoscopic surgery. Postoperative pain has been reported to cause elevated blood pressure [6]. Since hypertension is considered a risk factor for cardiac rupture after acute myocardial infarction [7], good control of postoperative pain may contribute to a reduced risk of cardiac rupture. In this case, opioids were used for analgesia, with typical doses, and the patient did not develop marked hypertension that required additional postoperative antihypertensive drugs. 
The third reason is that the increase in inflammatory cytokines, such as IL-1 $\beta$ and IL-6, is smaller in laparoscopic surgery than in open surgery. A study showed that laparoscopic surgery for RS rectal cancer resulted in a smaller increase in inflammatory cytokines (IL-1 $\beta$ and IL-6) and CRP (induced by these cytokines) compared with open surgery [8]. In addition, a report revealed that laparoscopic surgery is associated with a smaller increase in IL-6 compared to open surgery [9]. Inflammatory cytokines have been reported to increase the risk of acute heart failure and lethal ventricular arrhythmias, which are cardiac complications of acute myocardial infarction $[2,3]$. Therefore, by selecting laparoscopic surgery in which the increase in inflammatory cytokines is relatively suppressed, it is possible to suppress cardiac complications such as acute heart failure and fatal ventricular arrhythmia.

The fourth reason is that the postoperative intestinal function recovers quickly. Postoperative intestinal function is said to be restored faster in laparoscopic surgery than in open surgery [4]. If the intestinal function recovers early, it is possible to promptly resume oral intake and medications. In our case, all drugs including clopidogrel could be resumed orally from POD1.

The limitation of this case report is that there was only one case, without comparative cases. Therefore, there is no strong evidence of the superiority of laparoscopic surgery over open surgery immediately after acute myocardial infarction. However, accumulating a large number of similar cases is difficult, and it is necessary to consider through such case reports.

For oncological emergencies due to bleeding from colorectal cancer that occur immediately after acute myocardial infarction, laparoscopic surgery may be an excellent method in terms of preventing intraoperative complications and postoperative cardiac complications.

\section{Declarations}

Ethics declarations

\section{Ethics approval and consent to participate}

Written informed consent was obtained from the patient for participation. A copy of the written consent is available for review by the Editor-in-Chief of this journal.

\section{Consent for publication}

Written informed consent was obtained from the patient for publication of this case report and any accompanying images. A copy of the written consent is available for review by the Editor-in-Chief of this journal.

\section{Competing interests}

The authors declare that they have no competing interests.

\section{Funding}


Not received.

\section{Authors' contributions}

YT evaluated the patient clinically and write and revised the paper. YK, TH and KH evaluated the patient clinically and read and revised the paper. All authors read and approved the final manuscript.

\section{Acknowledgements}

We express our gratitude to the patient who kindly gave consent for this case to be presented in this article.

\section{Funding}

Not received.

\section{References}

1. Illuminati G, Ceccanei G, Pacilè MA, Pizzardi G, Palumbo P, Vietri F. Dual antiplatelet treatment in patients candidates for abdominal surgery. Ann Ital Chir. 2013;84: 291-294.

2. Suleiman M, Khatib R, Agmon Y, Mahamid R, Boulos M, Kapeliovich M, et al. Early inflammation and risk of long-term development of heart failure and mortality in survivors of acute myocardial infarction predictive role of C-reactive protein. J Am Coll Cardiol. 2006;47: 962-968.

3. Francis Stuart SD, De Jesus NM, Lindsey ML, Ripplinger CM. The crossroads of inflammation, fibrosis, and arrhythmia following myocardial infarction. J Mol Cell Cardiol. 2016;91: 114-122.

4. Veldkamp R, Kuhry E, Hop WCJ, Jeekel J, Kazemier G, Bonjer HJ, et al. Laparoscopic surgery versus open surgery for colon cancer: short-term outcomes of a randomised trial. Lancet Oncol. 2005;6: 477-484.

5. Fujikawa T, Ando K. Safety of laparoscopic surgery in digestive diseases with special reference to antithrombotic therapy: a systematic review of the literature. World J Clin Cases. 2018;6: 767-775.

6. Haas CE, LeBlanc JM. Acute postoperative hypertension: a review of therapeutic options. Am J Health Syst Pharm. 2004;61: 1661-1673; quiz 1674-5.

7. Wehrens XHT, Doevendans PA. Cardiac rupture complicating myocardial infarction. Int J Cardiol. 2004;95: 285-292.

8. Leung KL, Lai PB, Ho RL, Meng WC, Yiu RY, Lee JF, et al. Systemic cytokine response after laparoscopic-assisted resection of rectosigmoid carcinoma: a prospective randomized trial. Ann Surg. 2000;231: 506-511. 
9. Sammour T, Kahokehr A, Zargar-Shoshtari K, Hill AG. A prospective case-control study of the local and systemic cytokine response after laparoscopic versus open colonic surgery. J Surg Res. 2012;173: 278-285.

\section{Figures}

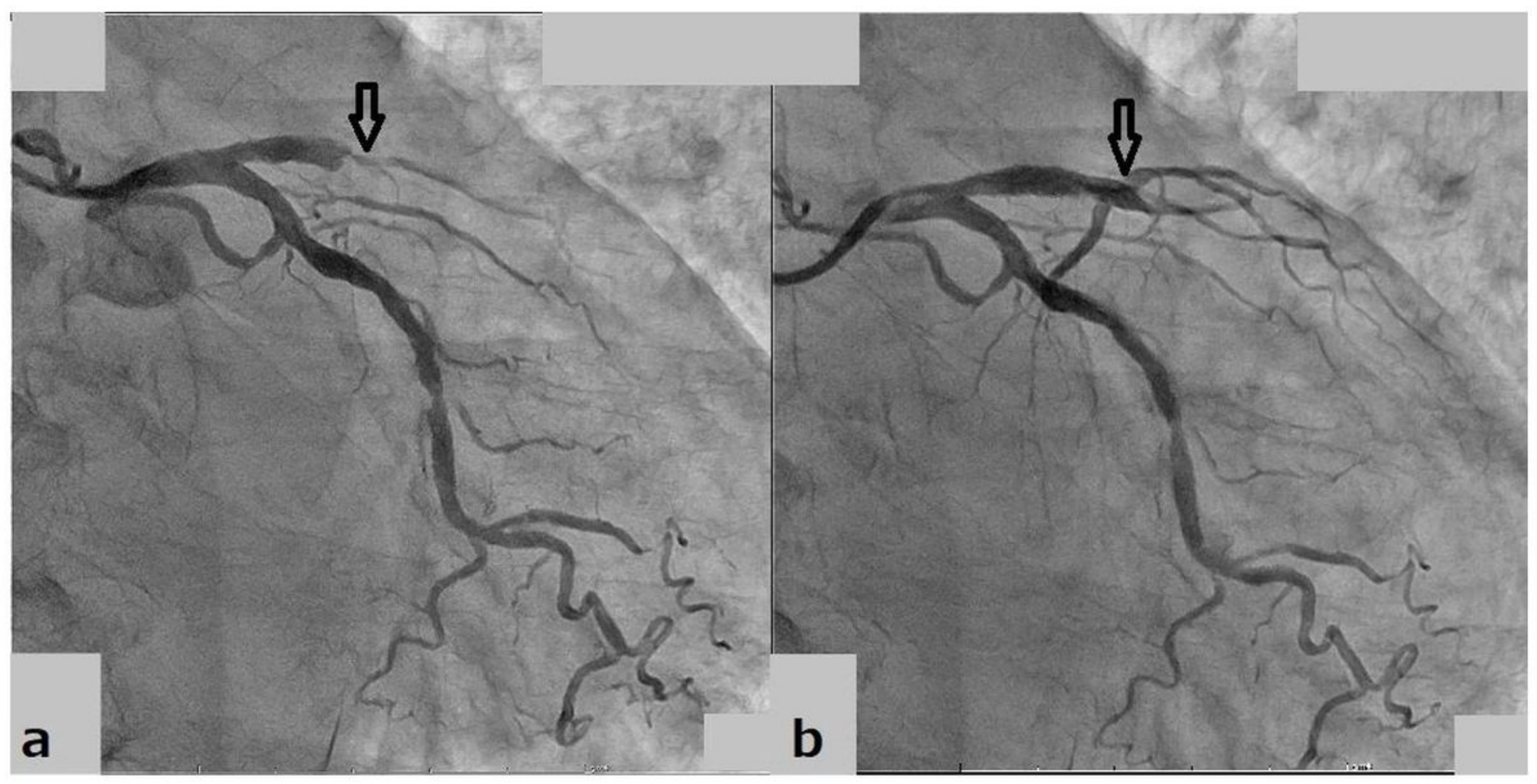

\section{Figure 1}

Coronary angiography image and percutaneous coronary intervention Coronary angiography showed \#6 $100 \%$ stenosis (a: arrow). Thus, PCl was performed on the same site, and a drug-eluting stent (Xience Skypoint) was placed; improvement from TIMI 0 to 3 was noted (b: arrow) 


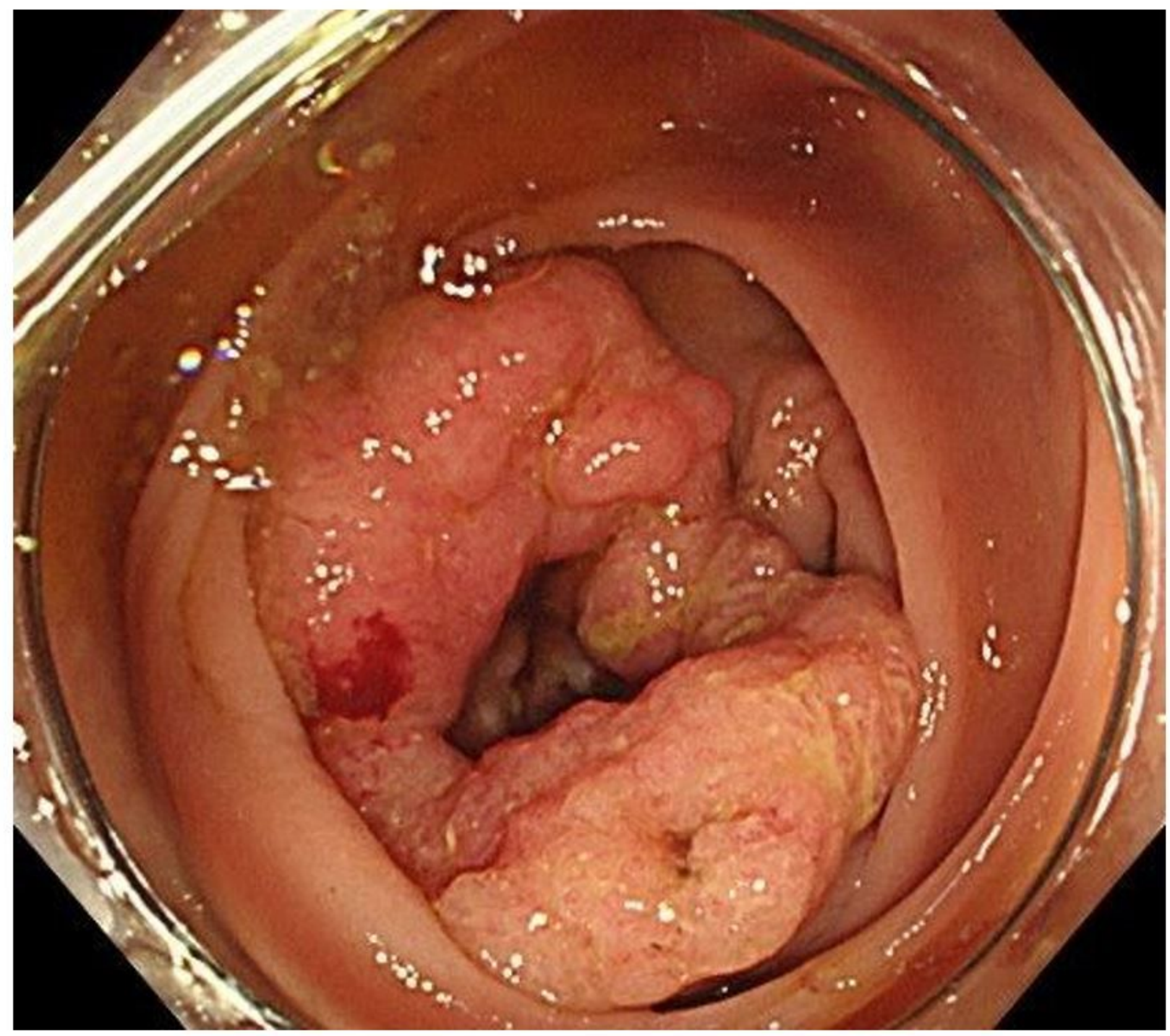

Figure 2

Lower endoscopy revealed a type 2 tumour of about $40 \mathrm{~mm}$ in the caecum, and biopsy revealed moderately differentiated adenocarcinoma, leading to a diagnosis of caecal cancer. 


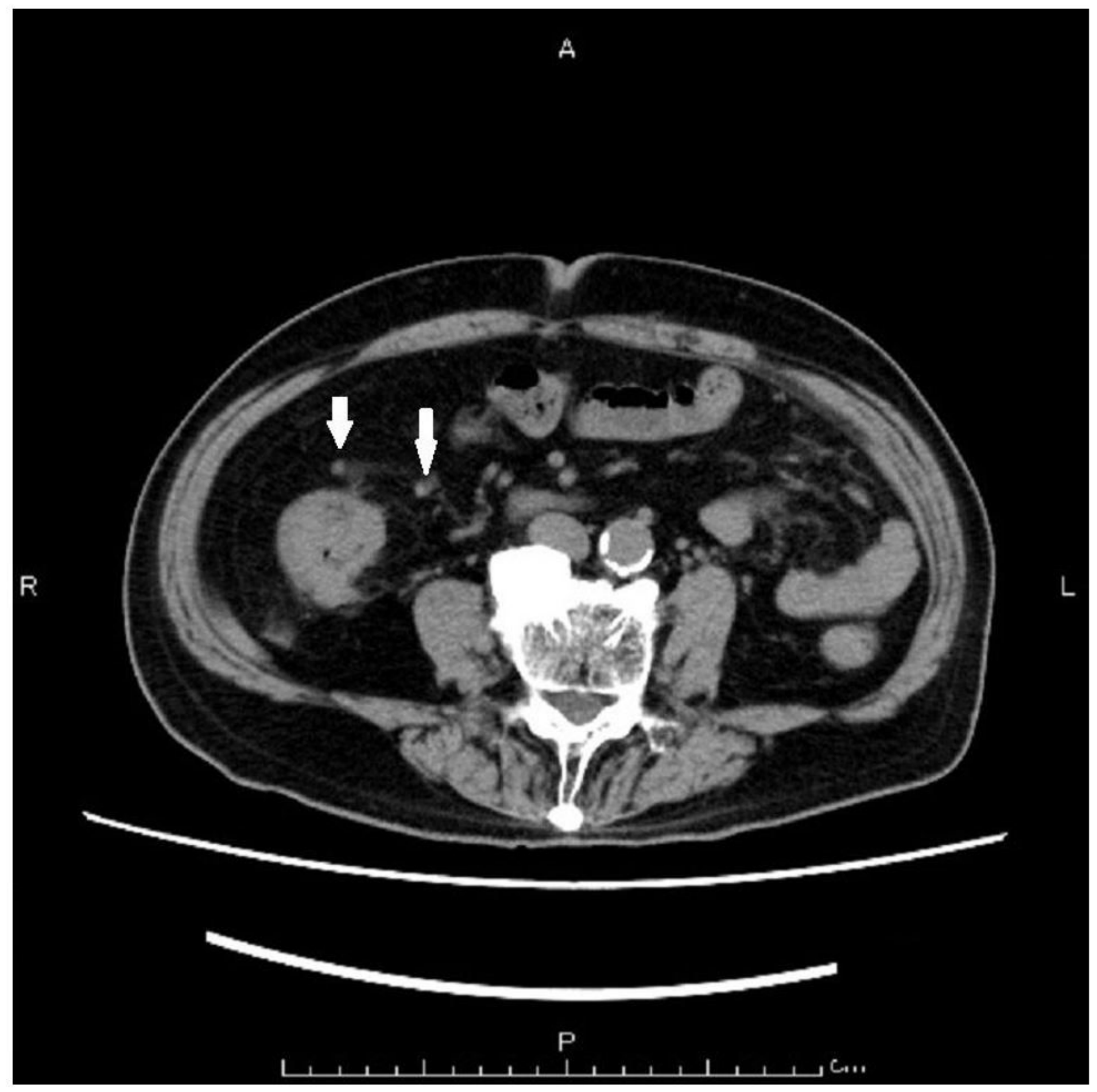

\section{Figure 3}

Computed tomography image Computed tomography showed swelling in the regional lymph node, which was suspected to be metastasis. 


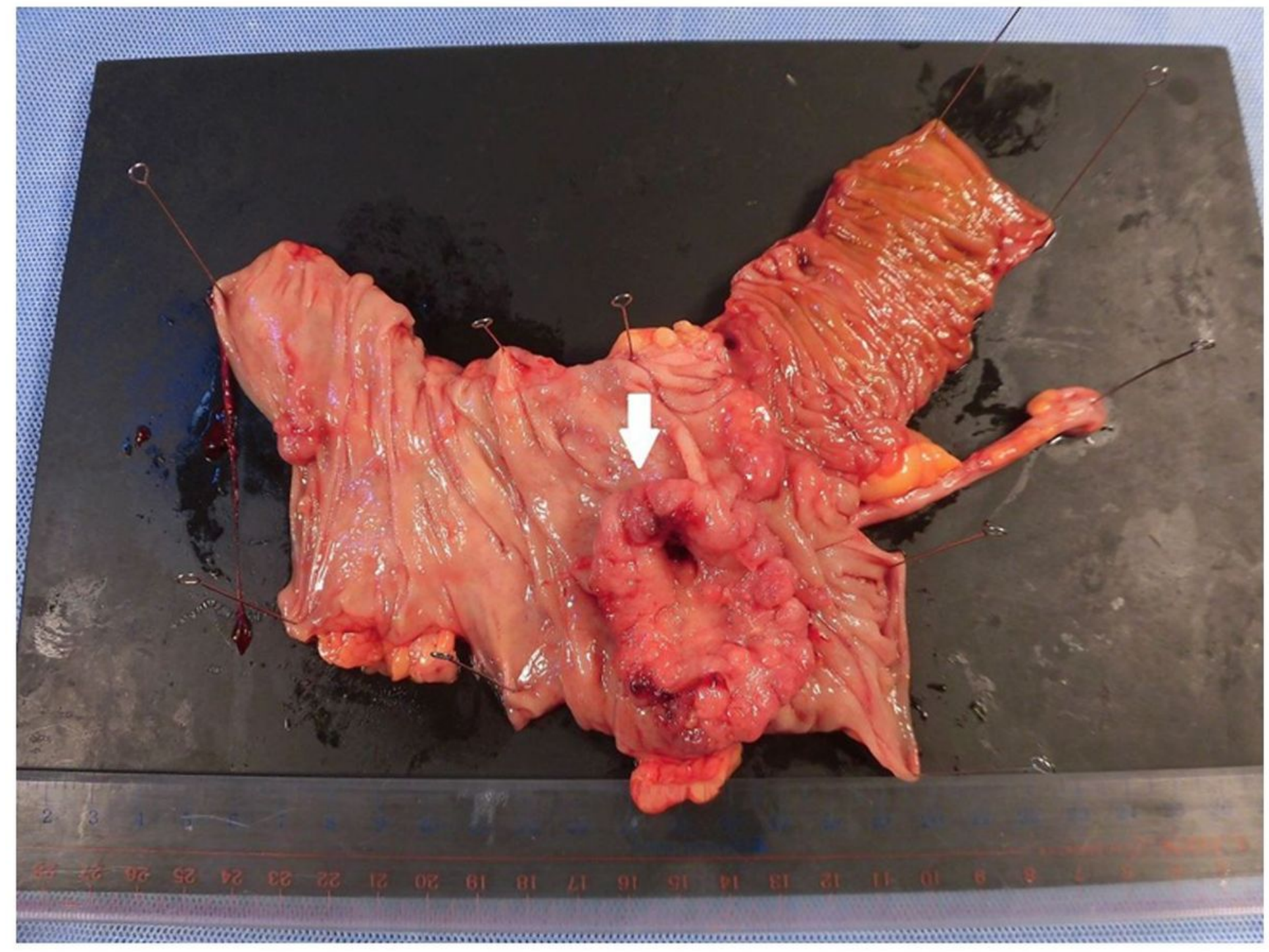

Figure 4

Image of the resected specimen Type 2 lesions were found in the resected caecum specimen as preoperatively diagnosed. 